\title{
Opening Injection Pressure Is Higher in Intraneural Compared With Perineural Injections During Simulated Nerve Blocks of the Lower Limb in Fresh Human Cadavers
}

\author{
Kris Vermeylen, $M D$, * Marlies Hermans, MD, † Filiep Soetens, MD, * Evie Vereecke, \\ Thorsten Steinfeldt, MD, PhD, § Gerbrand Groen, MD, PhD,// \\ Admir Hadzic, MD, PhD, I and Marc Van de Velde, MD, PhD\#
}

\begin{abstract}
Background and Objectives: Needle-induced nerve trauma and intraneural injection can lead to neurologic injury during peripheral nerve blocks. In this study, we assessed the utility of opening injection pressure (OIP), time to OIP, and rate of rise to OIP in detecting needle-nerve contact and intraneural injection.

Methods: Five common ultrasound-guided blocks of the femoral, saphenous, subgluteal sciatic, tibial, and common peroneal nerves were simulated in 10 fresh cadavers. Opening injection pressure was defined as peak psi in the 60-second interval during which the injection is initiated. Pressure-time curves were constructed separately for intraneural and perineural injections for each of the 5 nerves studied.

Results: Opening injection pressure was higher for intraneural than for perineural injections $(P<0.001)$, ranging from $21.5 \mathrm{psi}(1111.9 \mathrm{~mm} \mathrm{Hg})$ to $25.8 \mathrm{psi}(1334.2 \mathrm{~mm} \mathrm{Hg})$ for intraneural injections and from $3.8 \mathrm{psi}$ $(196.5 \mathrm{~mm} \mathrm{Hg})$ to $6.1 \mathrm{psi}(315.5 \mathrm{~mm} \mathrm{Hg})$ for perineural injections. Time to OIP tended to be shorter for intraneural than for perineural injections, particularly for the subgluteal sciatic, tibial, and common peroneal nerves. Curves of intraneural injections had steep slopes with high peaks; curves of perineural injections had low slopes followed by plateaus. Rise to OIP was greater for intraneural than for perineural injections.
\end{abstract}

Conclusions: In our fresh human cadaver model, OIP detected intraneural needle placement. Monitoring of OIP could be useful in detecting and/or preventing intraneural injection during nerve blocks in the clinical setting.

(Reg Anesth Pain Med 2017;42: 00-00)

From the *Department of Anaesthesia, AZ Turnhout, Turnhout; $\uparrow$ Department of Anaesthesia, University Hospital Antwerp, Antwerp; and †Department of Development and Regeneration, Jan Palfijn Anatomy Lab, University of Leuven Campus Kulak, Kortrijk, Belgium; §Department of Anaesthesia and Intensive Care, Philipps-University Hospital, Marburg, Germany; |Pain Centre, Department of Anesthesiology, University Medical Centre Groningen, University of Groningen, Groningen, the Netherlands; and $\mathbb{N}$ NAICE (North American Institute of Continuing Education) and Anesthesiology ZOL, Genk; and \#Department of Cardiovascular Sciences, KU Leuven and Department of Anaesthesiology, UZ Leuven, Leuven, Belgium.

Accepted for publication November 22, 2016.

Address correspondence to: Kris Vermeylen, MD, Department of Anesthesia, AZ Turnhout, Steenweg op Merksplas, 2300 Turnhout, Belgium (e-mail: kris.vermeylen@gmail.com).

A.H. has consulted and advised for Philipps, GE, SonoSite, Codman \& Shrutleff, Inc (Johnson and Johnson), Cadence, Pacira, Baxter, and B. Braun Medical. His recent industry-sponsored research includes work supported by Pacira Pharmaceuticals. A.H. receives royalty income from B. Braun Medical. A.H.'s role in this article involved scientific advice on the methodology as a mentor to a $\mathrm{PhD}$ project and manuscript drafting. A.H. did not participate and was not present during any study procedures and study execution. The other authors declare no conflict of interest.

K.V. received a grant to conduct this research from the Belgian Association of Regional Anesthesia in 2014.

Copyright (C) 2016 by American Society of Regional Anesthesia and Pain Medicine

ISSN: 1098-7339

DOI: 10.1097/AAP.0000000000000548
D espite ultrasound guidance, the risk of neurologic complications with peripheral nerve blocks remains unchanged. ${ }^{1-3}$ The reported incidence of complications, including local anesthetic toxicity and transient nerve injury, ${ }^{4-7}$ is relatively high. ${ }^{8,9}$ The reported incidence (per 1000 blocks) of adverse events across all peripheral regional anesthetics is 1.8 ( $95 \%$ confidence interval, 1.1-2.7); for postoperative neurologic symptoms (PONSs) lasting longer than 5 days, 0.9 (95\% confidence interval, $0.5-1.7)$, and for PONSs lasting longer than 6 months, $0.08 \%$. Needleinduced mechanical injury to the nerve and intraneural injection of local anesthetic have been reported in several studies to be the main factors. ${ }^{10}$ Recently, a Regional Anesthesia and Pain Medicine consensus statement proposed that earlier detection of AQ3 needle-to-nerve contact and avoidance of intraneural injection during peripheral nerve blocks might reduce the risk of nerve injury. ${ }^{11}$ Two studies monitored opening injection pressure (OIP), the pressure in the syringe-tubing-needle system at which an injection can commence at the tip of the needle. Both detected nearly $100 \%$ of the needle-nerve contacts in their patients and prevented injections against the nerve by aborting injection when OIP reached $15 \mathrm{psi}$ $(775.7 \mathrm{~mm} \mathrm{Hg}){ }^{12,13}$ Hence, if OIP, time to achieve OIP (TOIP), and rate of rise to OIP (ROIP) differ between intraneural injections and perineural injections, these measures may also be useful in detecting and preventing intraneural injection.

Using a fresh cadaver sciatic nerve block model, this study tested the difference in OIP between intraneural and perineural injections. Pressure-time curves were also evaluated to assess the rates of pressure change in reaching OIP between intraneural and perineural injections.

\section{METHODS}

This study was approved by the ethical review board of the University of Leuven (NH019 2015-09-01).

\section{Specimen Preparation}

Ten fresh human cadavers were obtained from the Human Body Donation Program at the University of Leuven in collaboration with the Jan Palfijn Anatomy Lab (Kortrijk, Belgium). Cadavers with external signs of previous extremity surgery were excluded. The sample included 7 adult males and 3 adult females (all whites). All cadavers were thawed at room temperature 48 hours prior to the experiments.

\section{Nerve Block Protocol}

Using a closed tissue model setup mimicking clinical practice, ultrasound-guided blocks of 5 commonly blocked nerves of the lower limb (femoral, saphenous, subgluteal sciatic, tibial, and common peroneal) were simulated. The femoral nerve was ultrasonically located by transverse view, close to the femoral crease and lateral to the femoral artery. The saphenous nerve was located by 
ultrasound using a transverse view on the anteromedial midthigh next to the femoral artery. The subgluteal sciatic nerve was approached transversely on the posterior buttock, between the ischial tuberosity and the greater trochanter. The tibial and common peroneal nerves were identified distal to the popliteal bifurcation.

Needles (8-cm, 22-gauge, Sonoplex Stim cannula; Pajunk Medizintechnologie, Geisingen, Germany) were placed using an ultrasound machine (Flex Focus 500, linear probe, 18-6 MHz, probe no 8870; BK Ultrasound, Peabody, Massachusetts). Needle tips were placed bilaterally in 2 positions: either $2 \mathrm{~mm}$ away from the nerve (perineural- left leg) or within the nerve (intraneuralright leg). As in earlier articles, an "intraneural" position of the needle was defined as placement of the needle tip beyond the epineurium into the nerve structure, to the extent visible on ultrasound. ${ }^{14-16}$ In this study, intraneural needle placement required concurrent visual observation of the needle within the nerve and nerve swelling upon injection. ${ }^{15,17}$ Nonetheless, ultrasound did not allow differentiation between extrafascicular and

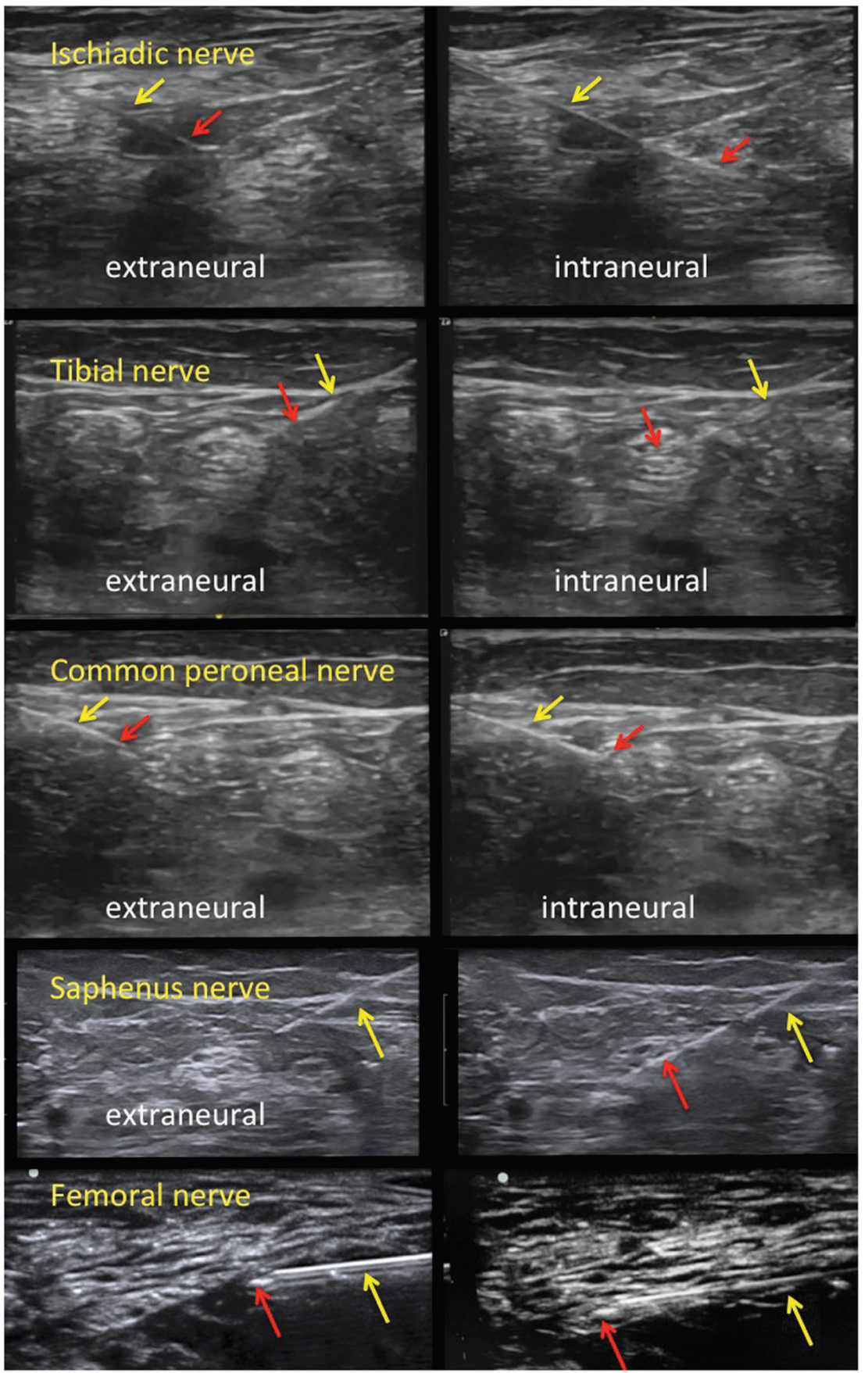

FIGURE 1. Overview of the 5 nerves with an extraneural approach and an intraneural needle placement. Arrows are needle in plane (red arrow in needle tip). 
intrafascicular placement of the needle. Each nerve received 1 F1 extraneural and 1 intraneural injection (Fig. 1). Thus, a total of 100 injections were performed during the study (50 intraneural F2 and 50 extraneural) (Fig. 2).

After needle placement, saline was delivered via an automatic infusion pump at $10 \mathrm{~mL} / \mathrm{min}$ to mimic clinical practice. $^{12}$ Injection pressure was continuously acquired using a digital pressure manometer, coupled to a computer for continuous pressure recording (Fluke IDA4, software program Hydrogragh; Fluke Biomedical Division of Fluke Electronics Corporation, Cleveland, Ohio). Injection pressure (psi) was assessed for 60 seconds after beginning the injection process with the pump. Prior to each injection, the manometer was calibrated to 0 (the basal injection pressure to air). As defined earlier, OIP was defined as pressure in the syringe-tubing-needle system at which injection can commence at the tip of the needle. Pressure-time curves were constructed separately for intraneural and perineural injections for each of the 5 nerves studied.

\section{Statistical Analysis}

Sample size for this study was estimated at 6 cadavers based on 2-sided $\alpha=0.05$, difference in psi important to detect pressure of $15 \mathrm{psi}(775.7 \mathrm{~mm} \mathrm{Hg}), \mathrm{SD}=3$, and power $=0.85$. Sample size was increased to 10 cadavers to accommodate the multiple nerves studied in the lower limb. Moreover, in order to keep the study- wise error rate at 0.05 , the aforementioned comparisons were Bonferroni corrected at $P=0.005(0.05 / 10)$. Statistical analyses were performed using the IBM SPSS Statistics for Windows, version 22.0 (IBM Corp, Armonk, New York).

Continuous measures are reported as mean $\pm \mathrm{SD}$; categorical (ordinal and nominal) measures as $\mathrm{n}(\%)$. To test whether OIP is higher with intraneural than with perineural injections, peak psi within the 60-second injection interval was compared between intraneural and perineural injections by $t$ tests for paired (dependent) samples for each of the 5 nerves studied. Time (in seconds) to reach OIP (TOIP) was compared between intraneural and perineural injections by $t$ tests for paired samples for each of the 5 nerves studied. Rates of ROIP (calculated as OIP/TOIP) for intraneural and perineural injections were also compared and were used to estimate a clinically relevant time to reach OIP for intraneural injections.

\section{RESULTS}

Opening injection pressure was higher for intraneural than for perineural injections for each nerve studied (Table 1). Time T1 to achieve OIP tended to be shorter for intraneural than for perineural injections, although reaching statistical significance only for the subgluteal sciatic nerve (Table 1).

For each of the 5 nerves, ROIP was higher for intraneural than for perineural injections (Table 1). For the femoral nerve,
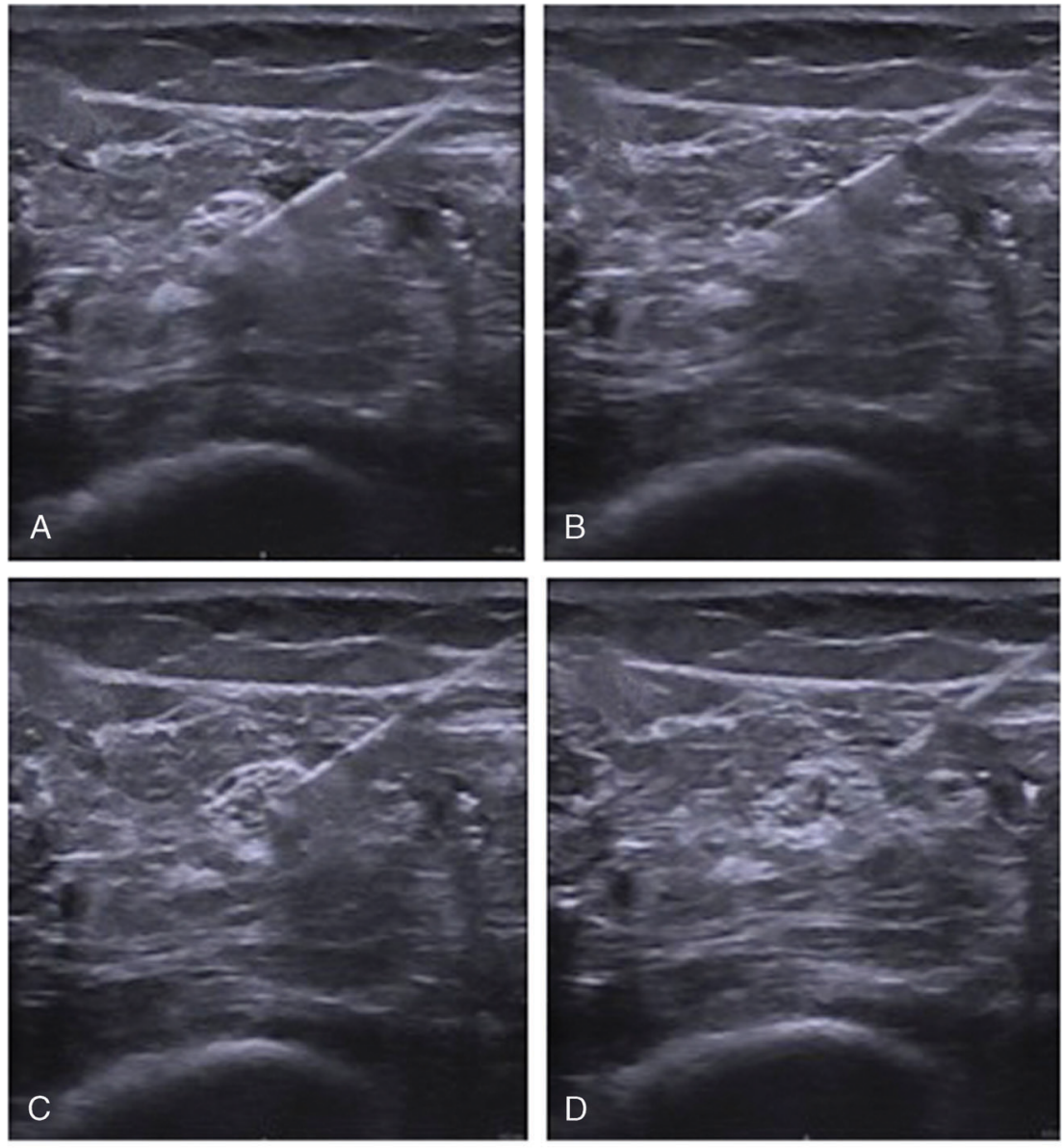

FIGURE 2. Intraneural injection of a tibial nerve. A, In-plane needle approach, (B) start injection, (C) swelling of the nerve, (D) rupture of the nerve with hypoechoic fluid (saline) within a predominantly hyperechoic neural structure. 
TABLE 1. OIP, TOIP, and Rate of ROIP for 5 Nerves of the Lower Limb Measured in 10 Fresh Frozen Cadavers

\begin{tabular}{|c|c|c|c|c|c|c|c|c|c|}
\hline \multirow[b]{2}{*}{ Nerve } & \multicolumn{3}{|c|}{ OIP (Peak psi) } & \multicolumn{3}{|c|}{ Time to Peak psi (s) } & \multicolumn{3}{|c|}{ Rate of Pressure Increase } \\
\hline & Intraneural & Perineural & $P^{*}$ & Intraneural & Perineural & $P^{*}$ & Intraneural & Perineural & $P^{*}$ \\
\hline Femoral & $22.4 \pm 4.7$ & $3.8 \pm 0.8$ & 0.005 & $21.8 \pm 6.8$ & $20.2 \pm 10.1$ & NS & $1.2 \pm 0.6$ & $0.2 \pm 0.1$ & 0.005 \\
\hline Saphenous & $21.7 \pm 2.6$ & $5.3 \pm 1.7$ & 0.005 & $20.0 \pm 8.0$ & $20.0 \pm 11.4$ & NS & $1.5 \pm 0.6$ & $0.3 \pm 0.2$ & 0.005 \\
\hline Subgluteal sciatic & $24.6 \pm 4.9$ & $4.5 \pm 2.2$ & 0.005 & $21.6 \pm 4.7$ & $39.2 \pm 13.4$ & 0.008 & $1.3 \pm 0.7$ & $0.3 \pm 0.4$ & 0.005 \\
\hline Tibial & $21.5 \pm 4.9$ & $6.1 \pm 1.9$ & 0.005 & $20.4 \pm 9.3$ & $30.8 \pm 17.1$ & 0.074 & $1.3 \pm 0.8$ & $0.4 \pm 0.4$ & 0.007 \\
\hline Common peroneal & $25.8 \pm 4.3$ & $5.6 \pm 1.7$ & 0.005 & $19.0 \pm 6.1$ & $29.2 \pm 18.3$ & NS & $1.2 \pm 0.4$ & $0.2 \pm 0.1$ & 0.005 \\
\hline
\end{tabular}

Data are pressure (in psi) mean \pm SD.

* $P$ values from Wilcoxon signed rank tests.

NS indicates not statistically significant.

ROIP was 6 times higher for intraneural than for perineural injections and was at least 3 times higher for the other nerves studied. Assuming injection rates similar to those used in standard clinical practice $(10 \mathrm{~mL} / \mathrm{min})$, pressure readings that reach the commonly used cutoff of 15 psi $(775.7 \mathrm{~mm} \mathrm{Hg})$ within 10 to 12 seconds of the start of the injection might be cautionary for intraneural needle position.

Curves of intraneural injections had a steep slope with a high peak; curves of perineural injections had low slope followed by a plateau. When the injections were stopped, the curves returned to 0 psi (Fig. 3).

\section{DISCUSSION}

In our fresh frozen cadaver model, intraneural injections in major nerves of the lower extremities resulted in several-fold higher OIPs than perineural injections. Similar to recent findings by other investigators, none of the perineural injections resulted
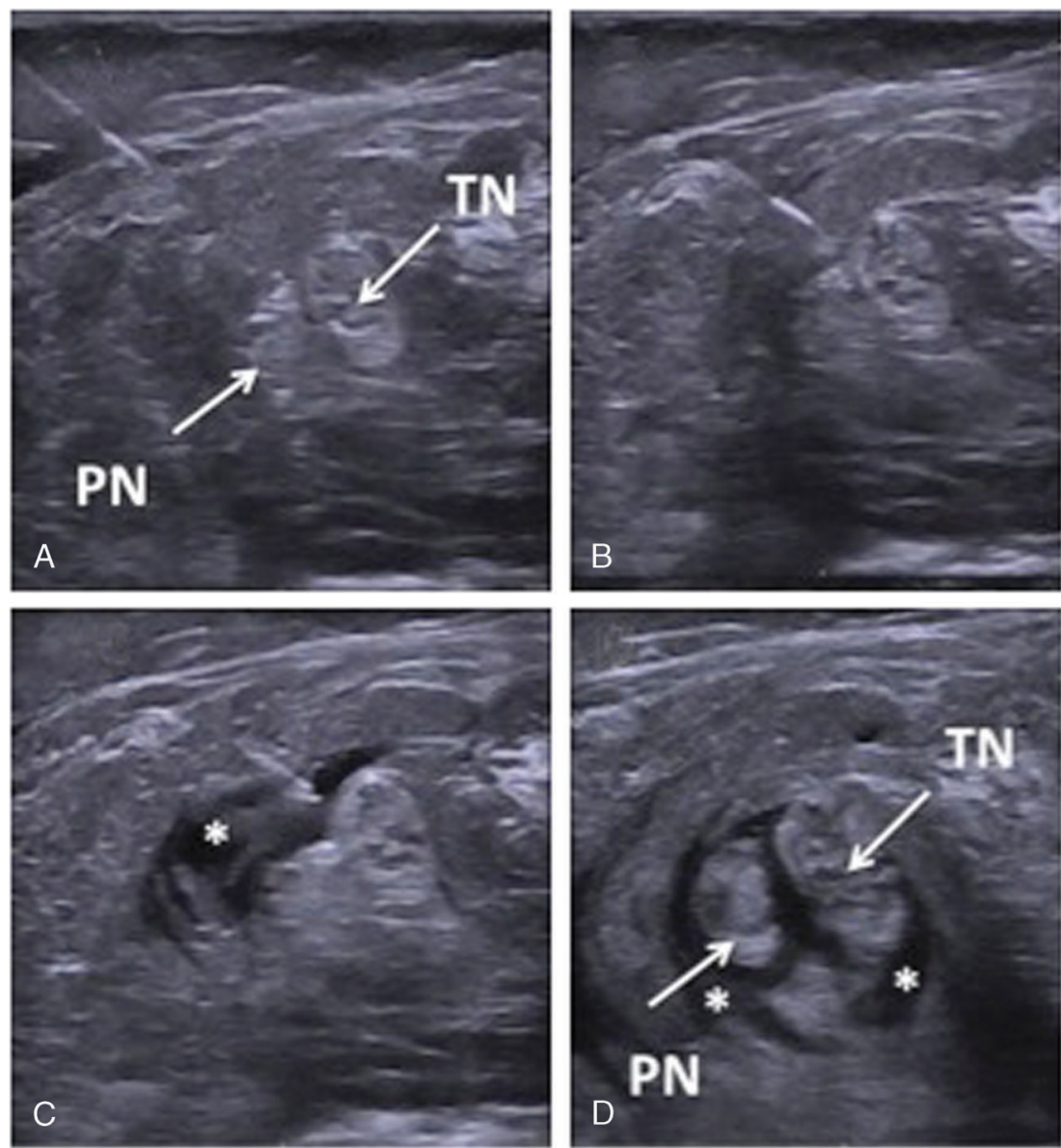

FIGURE 3. Extraneural injection of a popliteal nerve. A, Needle approach to the tibial and peroneal (popliteal) nerves, (B) start injection, (C) start saline injection, (D) saline around the popliteal nerve. TN indicates tibial nerve; PN, common peroneal nerve. *Injected local anesthetic. 


\section{Pressure-time}

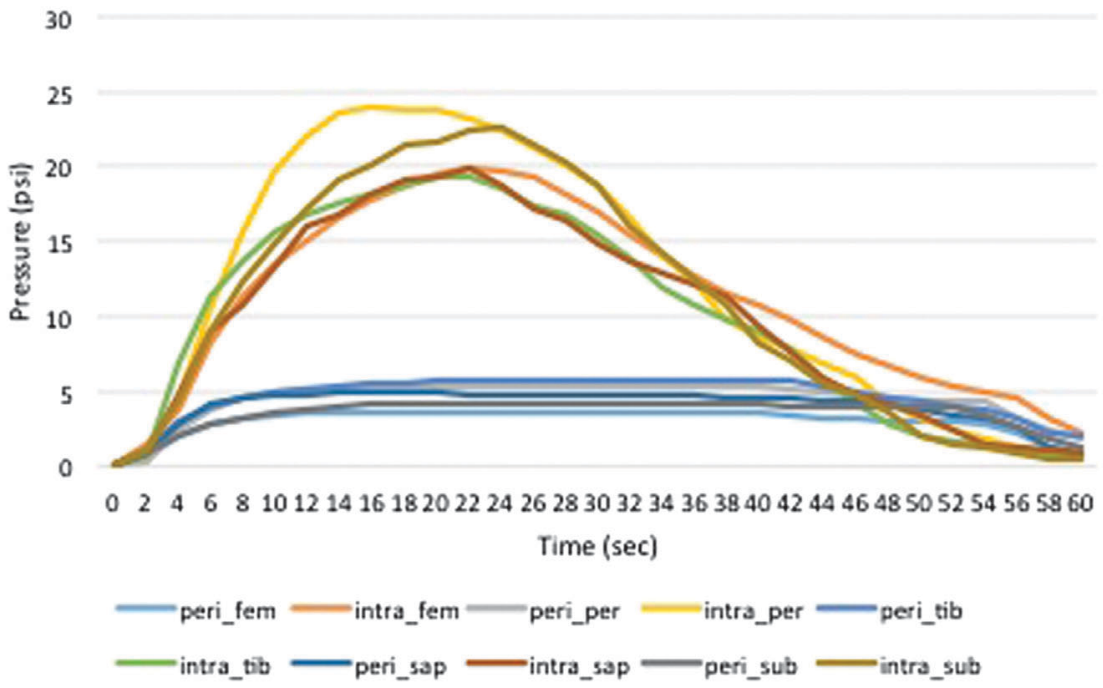

FIGURE 4. Pressure-time curves of the 5 nerves of the lower limb by type of injection (perineural and intraneural).

in OIPs greater than 15 psi, suggesting that this commonly used threshold for injection pressure may be applicable to lowerextremity nerve blocks as well. ${ }^{18,19}$ An interesting finding was that the rate of rise of OIP after beginning the injection process was 3 to 5 times higher for intraneural than for perineural injections. Previous investigators have not reported the rate of rise of injection pressure, although several reports detailed the injection pressure curves for intraneural, perineural, and intrafascicular injections. ${ }^{9,20,21}$ The potential significance of this finding is that rate of rise of injection pressure may indicate placement of the needle into a dense tissue medium sooner than OIP itself. This is because OIP varies from medium to medium and may even vary among different

F4 nerves or among the same nerves in different patients (Fig. 4).

Because of differences in neural architecture, ${ }^{19}$ the current clinical adoption of the 15-psi cutoff as "abnormally high" appears to be based on an empirical summary of the available studies in which intrafascicular injections or needle-nerve contact are consistently associated with OIP of 15 psi or greater., ${ }^{9,22,23} \mathrm{Be}-$

AQ4 cause we used the same system as did Krol et al. ${ }^{23}$ (with calibration before every measurement), our results may have also been due to differences in viscoelasticity in these nerves. An arbitrary pressure cutoff is rather nonspecific or may be detected too late in the cycle of the injection to prevent intraneural injection. In contrast, if the pattern of rate of rise of injection pressure seen in our study is replicable in other nerves (eg, of the upper extremity) and/ or in clinical patients, the ROIP may detect potential needle-nerve contact or intraneural injection sooner than reliance on an arbitrary OIP cutoff value.

\section{Limitations}

An important limitation of this study is the use of cadaveric material. We chose to conduct our study on fresh, unpreserved specimens because there is no literature on the effect of preservation method of measuring pressures in human body tissue. One study concluded that the use of Thiel's embalmed cadavers offers safer and more realistic conditions in the training and investigation of ultrasound-guided punctures, compared with fresh human cadavers. ${ }^{24}$ However, that study was based on the "feeling" of experienced anesthesiologists and did not use objective measurements of needle placement. Although it is possible that freezing the cadavers produced ice crystals, potentially increasing tissue rigidity, there is no reason to believe that the ice crystals would have differentially affected the pressure in intraneural and perineural tissue. An automatic infusion pump (rate, $10 \mathrm{~mL} / \mathrm{min}$ ) was chosen, similar to other studies and current best practice. In real life, however, some anesthesiologists might inject much faster and incrementally. The authors are convinced that a higher injection rate will not change the pressure rise differential.

\section{CONCLUSIONS}

This cadaver study suggests that OIP can be used to detect intraneural needle placement in lower-extremity peripheral nerves in fresh frozen human cadavers and therefore might prevent an intraneural injection by limiting the injection to an empirical value of less than 15 psi. The rate of rise of injection pressure, however, may be a more sensitive and earlier predictor of needle placement in dense tissue medium. Future studies are indicated to determine whether the pattern observed in our study is reproducible in other nerves and in living patients.

\section{ACKNOWLEDGMENT}

The authors thank Dr Bie Gielis for her assistance in performing the cadaver studies and Dr Max Kuroda for statistical analysis and editing the manuscript.

\section{REFERENCES}

1. Lee LA, Posner KL, Kent CD, Domino KB. Complications associated with peripheral nerve blocks: lessons from the ASA Closed Claims Project. Int Anesthesiol Clin. 2011;49:56-67.

2. Jeng CL, Torrillo TM, Rosenblatt MA. Complications of peripheral nerve blocks. Br J Anesth. 2010;105(suppl 1):i97-107.

3. Barrington MJ, Watts SA, Gledhill SR, et al. Preliminary results of the Australasian Regional Anaesthesia Collaboration: a prospective audit of more than 7000 peripheral nerve and plexus blocks for neurologic and other complications. Reg Anesth Pain Med. 2009;34:534-541.

4. Hogan QH. Pathophysiology of peripheral nerve injury during regional anesthesia. Reg Anesth Pain Med. 2008;33:435-441. 
5. Steinfeldt T, Wiesmann T. Needle-nerve proximity during peripheral nerve blocks - where is the right target and how can we get there? Rev Esp Anestesiol Reanim. 2015;62:121-4

6. Steinfeldt T, Poeschl S, Nimphius W, et al. Forced needle advancement during needle-nerve contact in a porcine model: histological outcome. Anesth Analg. 2011;113:417-20.

7. Cohen JM, Gray AT. Functional deficits after intraneural injection during interscalene block. Reg Anesth Pain Med. 2010;35:397-399.

8. Sites BD, Taenzer AH, Herrick MD, et al. Incidence of local anesthetic systemic toxicity and postoperative neurologic symptoms associated with 12,668 ultrasound-guided nerve blocks: an analysis from a prospective clinical registry. Reg Anesth Pain Med. 2012;37:478-482.

9. Orebaugh SL, Mukalel JJ, Krediet AC, et al. Brachial plexus root injection in a human cadaver model: injectate distribution and effects on the neuraxis. Reg Anesth Pain Med. 2012;37:525-529.

10. Farber SJ, Saheb-Al-Zamani M, Zieske L, et al. Peripheral nerve injury after local anesthetic injection. Anesth Analg. 2013;117:731-739.

11. Neal JM, Brull R, Chan VW, et al. The ASRA evidence-based medicine assessment of ultrasound-guided regional anesthesia and pain medicine: executive summary. Reg Anesth Pain Med. 2010;35:S1-S9.

12. Claudio R, Hadzic A, Shih H, et al. Injection pressures by anesthesiologists during simulated peripheral nerve block. Reg Anesth Pain Med. 2004;29: 201-205.

13. Sala-Blanch X, Ribalta T, Rivas E, et al. Structural injury to the human sciatic nerve after intraneural needle insertion. Reg Anesth Pain Med. 2009; 34:201-205.

14. Gadsden J, McCally C, Hadzic A. Monitoring during peripheral nerve blockade. Curr Opin Anaesthesiol. 2010;23:656-661.

15. Gadsden J. Injection pressure monitoring: no more excuses. J Clin Monit Comput. 2015;29:671-672.
16. Andersen HL, Andersen SL, Tranum-Jensen J. Injection inside the paraneural sheath of the sciatic nerve: direct comparison among ultrasound imaging, macroscopic anatomy, and histologic analysis. Reg Anesth Pain Med. 2012;37:410-414.

17. Moayeri N, Krediet AC, Welleweerd JC, Bleys RL, Groen GJ. Early ultrasonographic detection of low-volume intraneural injection. $\mathrm{Br} J$ Anaesth. 2012;109:432-438.

18. Krol A, Szarko M, Vala A, De Andres J. Pressure monitoring of intraneura an perineural injections into the median, radial, and ulnar nerves; lessons from a cadaveric study. Anesth Pain Med. 2015;5:e22723.

19. Gadsden JC, Choi JJ, Lin E, Robinson A. Opening injection pressure consistently detects needle-nerve contact during ultrasound-guided interscalene brachial plexus block. Anesthesiology. 2014;120: 1246-1253.

20. Selander D, Sjostrand J. Longitudinal spread of intraneurally injected local anesthetics. An experimental study of the initial neural distribution following intraneural injections. Acta Anaesthesiol Scand. 1978;22: $622-634$

21. Hadzic A, Dilberovic F, Shah S, et al. Combination of intraneural injection and high injection pressure leads to fascicular injury and neurologic deficits in dogs. Reg Anesth Pain Med. 2004;29:417-423.

22. Orebaugh SL. In ultrasound-guided interscalene block, do we know where the epineurium is? Anesthesiology. 2008;109:752-3. author reply 3.

23. Gadsden J, Latmore M, Levine DM, Robinson A. High opening injection pressure is associated with needle-nerve and needle-fascia contact during femoral nerve block. Reg Anesth Pain Med. 2016;41:50-55.

24. Benkhadra M, Faust A, Ladoire S, et al. Comparison of fresh and Thiel's embalmed cadavers according to the suitability for ultrasound-guided regional anesthesia of the cervical region. Surg Radiol Anat. 2009;31: $531-535$. 


\section{AUTHOR QUERIES}

\section{AUTHOR PLEASE ANSWER ALL QUERIES}

AQ1 = Please check if authors name are correctly captured for given names (in red) and surnames (in blue) for indexing after publication.

$\mathrm{AQ} 2$ = Please indicate academic degree(s) of author Vereecke.

AQ3 = "RAPM" was changed to "Regional Anesthesia and Pain Medicine," but is it the journal "Regional Anesthesia and Pain Medicine" or the society "American Society of Regional Anesthesia and Pain Medicine" that is meant? Please check.

$\mathrm{AQ} 4=\mathrm{Krol}$ is $\mathrm{nt}$ the author in Reference 23. Please check.

AQ5 = "P" was changed to "pressure." Please check if correct.

\section{END OF AUTHOR QUERIES}

